\title{
Circadian locomotor output cycles kaput affects the proliferation and migration of breast cancer cells by regulating the expression of E-cadherin via IQ motif containing GTPase activating protein 1
}

\author{
XIAOXUE LI ${ }^{1 *}$, SIYANG WANG ${ }^{2 *}$, SHUHONG YANG $^{1}$, JUNJIE YING ${ }^{1}$, \\ HANG YU ${ }^{1}$, CHUNLEI YANG $^{2}$, YANYOU LIU ${ }^{1}$, YUHUI WANG ${ }^{1}$, SHUTING CHENG $^{1}$, \\ JING XIAO $^{1}$, HUILING GUO ${ }^{1}$, ZHOU JIANG ${ }^{1}$ and ZHENGRONG WANG ${ }^{1}$ \\ ${ }^{1}$ Health Ministry Key Laboratory of Chronobiology, College of Basic Medicine and Forensic Medicine; \\ ${ }^{2}$ Department of Life Sciences, Sichuan University, Chengdu, Sichuan 610041, P.R. China
}

Received January 22, 2017; Accepted January 19, 2018

DOI: $10.3892 / \mathrm{ol} .2018 .8226$

\begin{abstract}
The circadian rhythm regulates numerous physiological activities, including sleep and wakefulness, behavior, immunity and metabolism. Previous studies have demonstrated that circadian rhythm disorder is associated with the occurrence of tumors. Responsible for regulating a number of functions, the Circadian locomotor output cycles kaput (Clock) gene is one of the core regulatory genes of circadian rhythm. The Clock gene has also been implicated in the occurrence and development of tumors in previously studies. The present study evaluated the role of the Clock gene in the proliferation and migration of mouse breast cancer $4 \mathrm{~T} 1$ cells, and investigated its possible regulatory pathways and mechanisms. It was reported that downregulation of Clock facilitated the proliferation and migration of breast cancer cells. Further investigation revealed the involvement of IQ motif containing GTPase activating protein 1 (IQGAP1) protein expression in the Clock regulatory pathway, further influencing the expression of E-cadherin, a known proprietor of tumor cell migration and invasion. To the best of our knowledge, the present study is the first to report that Clock, acting through the regulation of the scaffolding protein IQGAP1, regulates the downstream expression of E-cadherin, thereby affecting
\end{abstract}

\footnotetext{
Correspondence to: Professor Zhou Jiang or Professor Zhengrong Wang, Health Ministry Key Laboratory of Chronobiology, College of Basic Medicine and Forensic Medicine, Sichuan University, 9th Middle Segment, 17 Renmin South Road, Chengdu, Sichuan 610041, P.R. China

E-mail: wangzhengrong@126.com

E-mail: jiang-yuqian@126.com

*Contributed equally
}

Abbreviations: Clock, circadian locomotor output cycles kaput; IQGAP1, IQ motif containing GTPase activating protein 1

Key words: Clock, migration, proliferation, breast cancer tumor cell structure and motility. These results confirmed the role of Clock in breast cancer tumor etiology and provide insight regarding the molecular avenues of its regulatory nature, which may translate beyond breast cancer into other known functions of the gene.

\section{Introduction}

Circadian rhythms include all light-sensitive biological mechanisms that allow for the organismal alternations that occur with $24 \mathrm{~h}$. These include, but are not limited to, the regulation and control of the intra-corporal nerves, body fluids and immune systems. Regulation and control of biological circadian rhythms aids in maintaining the process of normal physiology, biochemistry and behaviors in organisms (1-3). In mammals, the hypothalamic suprachiasmatic nucleus is the major oscillator of circadian and biological rhythms. That same rhythm exists in peripheral tissues and cells under the control of the suprachiasmatic nucleus $(1,4,5)$. The intra-corporal mechanisms of biological rhythm control changes, including sleep, wakefulness, feeding, temperature and blood pressure, as well as physiological process, including cell cycle, DNA damage response, aging and metabolism. Biological rhythms, when disturbed, may lead to unbalanced biological functions and, in turn, the occurrence of diseases, including cardiovascular disease and tumors $(2,6,7)$.

Existing in the central suprachiasmatic nucleus and all peripheral tissues, circadian genes are the molecular mechanisms maintaining temporal rhythm in organisms. The biological circadian rhythm is regulated and maintained by the feedback loop formed by a group of genes, often featuring positive or negative regulation and control. Core circadian rhythm genes include positive regulating genes that activate the expression of rhythm genes (Bmall and Clock) and negative regulating genes that reduce the expression of rhythm genes, including Period (Per1, Per2 and Per3) and Cryptochrome (Cryl and Cry2) (3,7). Bmal1 and Clock proteins have the structural domain of transcription factor PAS-HLH, and positive regulation and control in the feedback loop. A Clock: Bmall heterodimer binds the CACGTG E-box 
enhancer regions of the $\mathrm{mPerl}, \mathrm{mPer} 2, \mathrm{mPer} 3, \mathrm{~m} C r y 1$ and mCry 2 genes; it drives the transcription and translation of several genes but produces $\mathrm{mPer}$ and $\mathrm{mCry}$, which are known inhibitors of the transcriptional activity of the Clock:Bmall heterodimer, thereby mediating the negative feedback loop. The mRNA and protein levels of the majority of clock genes generally exhibit 24-h cycle oscillation under the influence of their own feedback loops, but Clock is exceptional for its constitutive expression in tissue cells without any obvious fluctuations (8).

Biological rhythm disorder may have negative impacts on the physiological functions of mammals. A large amount of clinical and experimental evidence has demonstrated that biological rhythm disorder may lead to uncontrollable cell proliferation, implicating the disorder and its associated mechanisms in the etiology of cancer $(7,9,10)$. Breast cancer is the common malignant tumor among females, with 1.2 million women being diagnosed every year worldwide. Approximately 0.5 million women succumb to mortality as a result of breast cancer, making it the most dangerous of all malignant tumors in females. There are numerous risk factors for breast cancer, including menstruation, childbearing, a high-fat diet and a family history. Epidemiological reports have demonstrated that circadian rhythm disorder may increase the chance of females developing breast cancer (11-13). In general, cancer has been linked to rhythm disorder, but a detailed molecular mechanism has yet to be fully elucidated. The Clock gene is confirmed to be a core member of the circadian system, and has also been demonstrated to serve an important role in tumor growth. The present study aimed to investigate the effects of Clock on the proliferation and migration of breast cancer cells, in addition to elucidating the molecular mechanisms regulating the biological actions of the gene in a breast cancer cell line. It was reported that E-cadherin, under the regulation of the scaffolding protein, IQ motif containing GTPase activating protein 1 (IQGAP1), mediates the structural and mobility features that allow tumor cells to proliferate and become invasive. This pathway, regulated by the Clock gene, provides a clearer picture between circadian clock disorder and breast cancer, and lays the groundwork for future study.

\section{Materials and methods}

Cell culture and transfection. 4T1 cells were obtained from the American Type Culture Collection cell bank (Manassas, VA, USA). They were cultured in Dulbecco's modified Eagle's medium (Hyclone; GE Healthcare Life Sciences, Logan, UT, USA), containing $10 \%$ inactivated fetal bovine serum (Gibco; Thermo Fisher Scientific, Inc., Waltham, MA, USA). Subsequently, cells were maintained in cell incubators with $5 \% \mathrm{CO}_{2}$ at a constant temperature of $37^{\circ} \mathrm{C}$.

Lentiviral transfection. Mouse Clock small hairpin RNA(shRNA) was constructed in the lentivirus gene transfer vector pHBLV-U6-ZsGreen, and the titer of the virus was $2 \times 10^{8} \mathrm{TU} / \mathrm{ml}$. lv-shRNA-Clock and lv-GFP-Puro NC viruses containing a green fluorescent protein (GFP) sequence (Hanbio Biotechnology Co., Ltd., Zhejiang, China) were thawed and dissolved on ice. Once the medium was replaced with fresh medium containing the transfection reagent polybrene
(Hanbio Biotechnology Co., Ltd.), the virus solutions were added into the 24-well plate containing 4T1 cells at a volume of $30 \mu \mathrm{l} / \mathrm{well}$, according to the manufacturer's protocols. The cells were cultured in the incubator at a constant temperature of $37^{\circ} \mathrm{C}$ and a saturation humidity of $5 \% \mathrm{CO}_{2}$ for $24 \mathrm{~h}$. At $48 \mathrm{~h}$ post-transfection, cells were detected using a fluorescence microscope (magnification, x 100) (Nikon Corporation, Tokyo, Japan). Expression efficiency of the vectors was assessed using GFP. Subsequently, the cells were transferred to cell culture bottles where they were screened for successful transfection using puromycin (J\&K Scientific Ltd., Beijing, China) at a concentration of $2 \mu \mathrm{g} / \mathrm{ml}$, according to the manufacturer's protocols. The cycle for transfection screening was one week.

Cell proliferation assay. With a total volume of $100 \mu \mathrm{l}$ nutrient solution, the cells screened out for successful transfection were inoculated in 96-well plates at 1,000 cells/well. Next, the starting position of cell adherence was marked at 0 days. Cells were incubated with $10 \mu 1$ Cell Counting Kit-8 (CCK-8; Dojindo Molecular Technologies, Inc., Kumamoto, Japan) and placed in an incubator with a saturation humidity of $5 \% \mathrm{CO}_{2}$ at a constant temperature of $37^{\circ} \mathrm{C}$ for $1 \mathrm{~h}$. A microplate reader (Thermo Fisher Scientific, Inc.) was used to detect light absorption at $450 \mathrm{~nm}$ after 1, 2, 3, 4 and 5 days incubation.

Cells screened for successful transfection were inoculated in 6-well plates at 500 cells/well with a total volume of $2 \mathrm{ml}$ nutrient solution. Then cells were placed in the incubator with a saturation humidity of $5 \% \mathrm{CO}_{2}$ and a constant temperature of $37^{\circ} \mathrm{C}$ for 2 weeks. After 2 weeks, plates were washed with phosphate-buffered saline (PBS) 2-3 times and $1 \mathrm{ml}$ methyl alcohol was added for fixation for $\sim 30 \mathrm{~min}$ at room temperature. Following drying, $1 \mathrm{ml}$ crystal violet was added to each well for $\sim 2 \mathrm{~min}$ at room temperature.

Detection of cell cycle distribution with flow cytometry. A single-cell suspension was made by $1,000 \mathrm{x}$ g centrifugation at $4^{\circ} \mathrm{C}$ for $5 \mathrm{~min}$ of cells successfully transfected by the virus. Cells were centrifuged at $1,000 \mathrm{x}$ g at $4^{\circ} \mathrm{C}$ for $5 \mathrm{~min}$ and were washed three times with precooled PBS. A second centrifugation was performed at $1,000 \mathrm{xg}$ at $4^{\circ} \mathrm{C}$ for $5 \mathrm{~min}$. The cell sediment was resuspended with precooled $75 \%$ ethyl alcohol for cell fixation at $4^{\circ} \mathrm{C}$ for $12 \mathrm{~h}$, followed by staining with $50 \mu \mathrm{l}$ propidium iodide (PI; $0.4 \mathrm{mg} / \mathrm{ml}$; Sigma-Aldrich; Merck KGaA, Darmstadt, Germany) and $50 \mu \mathrm{l}$ RNase (Sigma-Aldrich; Merck KGaA). Incubation was performed for $30 \mathrm{~min}$ at $37^{\circ} \mathrm{C}$ in the dark. Subsequently, the percentage of cells in the G0/G1 phase and S phase, respectively, was calculated using a FACSCanto II flow cytometer (BD Biosciences, Franklin Lakes, NJ, USA) and ModFit LT software (Verity Software House, Inc., Topsham, ME, USA). Each experiment was repeated three times.

Cell migration assay. Serum-free Dulbecco's modified Eagle's medium (200 $\mu \mathrm{l}$; Hyclone; GE Healthcare Life Sciences) was added to $~ 50,000$ cells in the upper chamber of the Transwell filters (8.0- $\mu \mathrm{m}$ pores). Subsequently, $900 \mu \mathrm{l}$ Dulbecco's modified Eagle's medium, containing 20\% fetal bovine serum, was loaded into the lower chamber. Following inoculation, cells were cultured for $24 \mathrm{~h}$ at $37^{\circ} \mathrm{C}$, were washed three times with 
PBS and were fixed with $100 \%$ methyl alcohol for $10-15$ min at room temperature. Following drying, invaded cells on the bottom surface of the Transwell were stained with crystal violet (Sigma-Aldrich; Merck KGaA) for $30 \mathrm{sec}$ at room temperature, washed with PBS until the membrane was clear of dye. Next, the membrane was cut and observed under a light microscope (magnification, x100; Nikon, Japan). Data are presented as the mean \pm standard deviation of the number of the cells/field in 3 individual experiments.

Western blot analysis. Cells were collected during the logarithmic phase and were lysed with radioimmunoprecipitation assay buffer, containing $1 \mathrm{mM}$ PMSF, Halt protease inhibitor cocktail and Halt phosphatase inhibitor (Beyotime Institute of Biotechnology, Haimen, China) for $30 \mathrm{~min}$ on ice. The resolved proteins underwent centrifugation at $14,000 \mathrm{x} \mathrm{g}$ for $20 \mathrm{~min}$ at $4^{\circ} \mathrm{C}$. Protein concentrations were determined using a bicinchoninic acid protein assay (Beyotime Institute of Biotechnology). Equal amounts of protein $(0.5 \mathrm{mg} / \mathrm{ml}$ concentration; control and experimental) were loaded in each experiment. Protein was separated by $10-12 \%$ SDS-PAGE, followed by transfer to polyvinylidene difluoride membranes (Millipore, USA). The membrane was blocked with $5 \%$ skim milk for $2 \mathrm{~h}$ at room temperature, incubated with the following primary antibodies: Monoclonal anti- $\beta$-actin (1:5,000 dilution; cat no. 60008-1-Ig; ProteinTech Group, Inc., Chicago, IL, USA), anti-Clock (1:10,000 dilution; cat no. ab3517; Abcam, Cambridge, MA, USA), anti-cluster of differentiation (CD)44 (1:1,000 dilution; cat no. CY5138; Shanghai Abways Biotechnology Co., Ltd., Shanghai, China), anti-tumor protein p53 (1:1,000 dilution; cat no. AB3125; p53; Shanghai Abways Biotechnology Co., Ltd.), anti-E-cadherin (1:2,000 dilution; cat no. CY1155; Shanghai Abways Biotechnology Co., Ltd.), anti-cyclin D1 (1:1,000 dilution; cat no. CY5404; Shanghai Abways Biotechnology Co., Ltd.), anti-IQGAP1 (1:2,000 dilution; cat no. 22167-1-AP; ProteinTech Group, Inc.), anti-heat shock protein 27 (1:1,000 dilution; cat no. CY5934; Hsp27; Shanghai Abways Biotechnology Co., Ltd.) and anti-PCNA (1:2,000 dilution; cat no. BM3888; Boster Biological Technology, Pleasanton, CA, USA). The membrane was incubated overnight at $4^{\circ} \mathrm{C}$, followed by incubation with a horseradish peroxidase-conjugated anti-rabbit $\mathrm{IgG}$ or anti-mouse $\mathrm{IgG}$ secondary antibody (1:5,000 dilution; cat no. SA00001-2\&SA00001-1; ProteinTech Group, Inc.) at $37^{\circ} \mathrm{C}$ for $2 \mathrm{~h}$. Membranes were developed using an enhanced chemiluminescence reagent from the EasySee Western Blot kit (Beijing Transgen Biotech Co., Ltd., Beijing, China) and imaged using a gel imager (GE Healthcare Life Sciences, Little Chalfont, UK). The images were further analyzed with ImageJ software (National Institutes of Health, Bethesda, MD, USA) to assess the differences in protein expression.

Statistical analysis. Data are presented as the mean \pm standard error of the mean and were analyzed by one-way analysis of variance followed by the Student-Newman-Keuls post hoc test. All data were averaged from three independent assays and were analyzed using GraphPad Prism 5.0 (GraphPad Software, Inc., La Jolla, CA, USA and SPSS 19.0 statistics software (IBM Corp., Armonk, NY, USA). P<0.05 was considered to indicate a statistically significant difference.

\section{Results}

Clock expression was efficiently decreased in $4 T 1$ cells. Stably-transfected 4T1 cells were named as follows: Control group (untransfected 4T1 cells), NC group (4T1 cells transfected with lv-GFP-Puro NC virus) and SC group (4T1 cells transfected with lv-shRNA-Clock virus). Observation of green fluorescence indicated that the number of cells successfully transfected was $>90 \%$ (Fig. 1A). Western blot analysis further demonstrated that the expression level of Clock protein in the $\mathrm{SC}$ group was significantly lower than that in the control and $\mathrm{NC}$ groups $(\mathrm{P}<0.05$; Fig. $1 \mathrm{~B}$ and $\mathrm{C})$.

Effects of Clock on cell proliferation. 4T1 cell lines knocked down by Clock-shRNA and control vectors were analyzed using a CCK-8 cell proliferation assay for 6 consecutive days. Data were used to form a longitudinal proliferation curve. Results demonstrated that the proliferation ability of cells knocked down by Clock-shRNA was enhanced compared with that of cells in the control group $(\mathrm{P}<0.05$; Fig. $2 \mathrm{~A})$. The monoclonal cell mass that had formed in the 6-well plates was dyed with crystal violet, which revealed that the monoclonal cell number in the SC group was significantly increased compared with cells in the control groups (Fig. 2B and C). Following further analysis of the cells by flow cytometry, cycle phases of the cells were determined using Modfit software (Verity Software House, Inc.). The cycle phase of the cells in the SC group was different to that of the control cells. When cells in the SC group were compared with those in $\mathrm{NC}$ group, the proportion of cells in the G0/G1 phase was lower, while the number of cells in the $\mathrm{S}$ phase increased $(\mathrm{P}<0.05$; Fig. $2 \mathrm{D}$ and $\mathrm{E})$. Western blot analysis revealed that the expression of cyclin D1, PCNA and p53 proteins was increased in the SC group (Fig. 3A and B), which further illustrated the enhanced proliferation ability of cells following Clock interference.

Effects of CLOCK on migration. A thin layer of membrane from the cells of each group were cut and fixed on a glass slide following fixation and crystal violet staining. Results demonstrated that the rate of migration of the cells in the SC group was significantly higher than that of the control cells (Fig. 4A and B). Furthermore, western blot analysis demonstrated that the expression level of E-cadherin in the SC group was similarly increased. E-cadherin is an important adherence factor on the surface of the cytomembrane, and has important functions in proliferation and migration of cells. The expression of IQGAP1 protein, which directly interacts with E-cadherin, was also markedly increased in the SC group compared with the control group. Finally, an increased expression of cell-migration-associated protein Hsp27 and membrane surface adhesion-associated protein CD44 was detected following Clock knockdown, compared with the control cells (Fig. 4C and D).

\section{Discussion}

In the present study, inhibition of clock gene expression was achieved in 4T1 cells by lentiviral transfection with Clock-shRNA. Cell proliferation and migration, and the expression of Clock-related proteins were assessed in a 
A
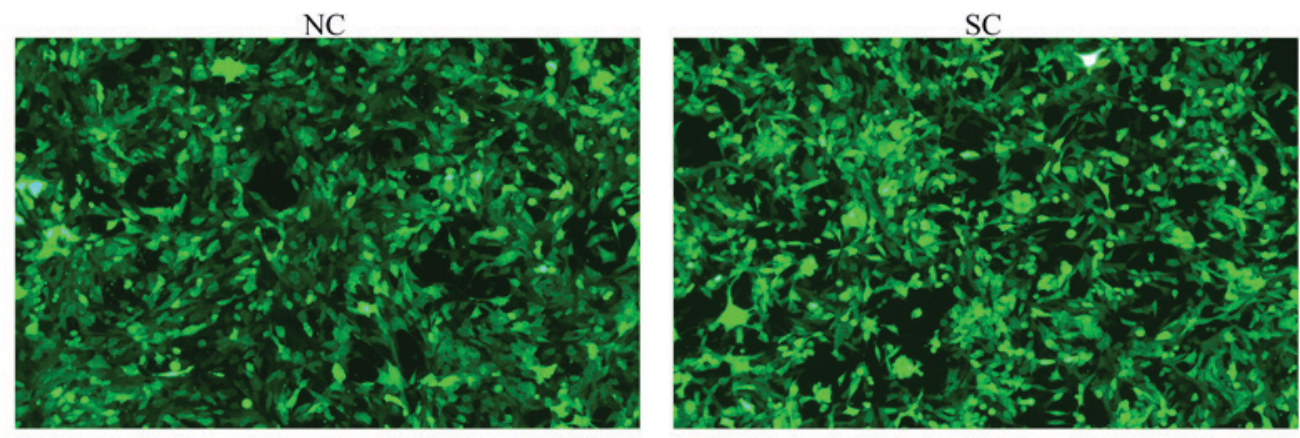

B

$\mathrm{C}$

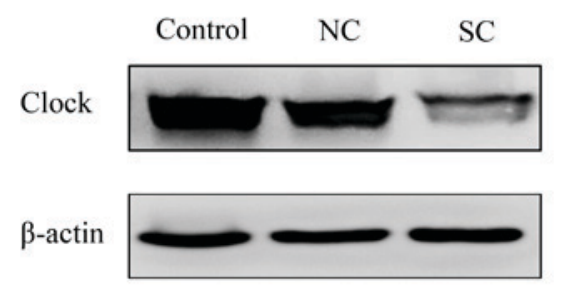

$95 \mathrm{kd}$

$42 \mathrm{kd}$

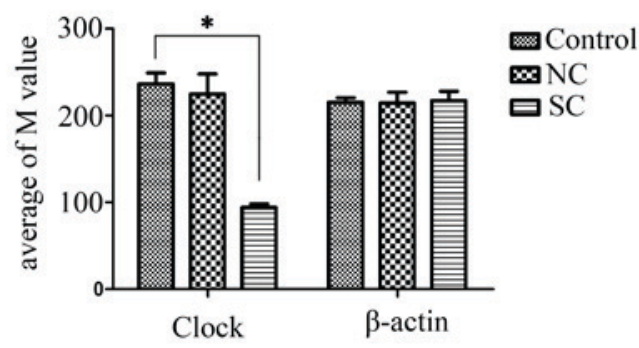

Figure 1. The expression of Clock in 4T1 cells. (A) Green fluorescence represents the transfection efficiency of the virus in the NC and SC groups (magnification, x100). (B) The protein expression of Clock was determined by western blot analysis. (C) Quantitation of the western blot analysis. Data are presented as the mean \pm standard deviation of three independent experiments. ${ }^{*} \mathrm{P}<0.05$ vs. control. Clock, circadian locomotor output cycles kaput; NC, negative control (4T1 cells transfected with lv-GFP-Puro NC virus); SC, 4T1 cells transfected with lv-shRNA-Clock virus.

breast cancer cell line in order to more clearly understand the association between circadian rhythm regulatory genes and the development of cancer. Following Clock downregulation, it was revealed that the proliferation of breast cancer cells improved, as determined by CCK- 8 and clone formation assays. Cell cycle distribution analysis using flow cytometry revealed that the number of cells in the $S$ phase was increased in the knockdown group, providing further evidence that the interference of Clock facilitates the proliferation of cells to a certain degree. This, coupled with the observed increase in migration of the knocked down cells, illustrates an endogenous inhibitory role of Clock on cellular proliferation and migration.

Cyclin D1, an important positive regulatory factor of the cell cycle, serves an important role in the occurrence and development of tumors $(14,15)$. Binding and activating the unique cyclin-dependent kinase, CDK4, during the G1 phase, cyclin D1 is responsible for the phosphorylation of retinoblastoma protein $(\mathrm{Rb})$ during the $\mathrm{G} 1$ phase (16). Rb protein is subsequently dissociated from its bound E2F transcription factors, which initiates gene transcription and activation of cell cycle genes, driving the cycle from the G1 phase to the $\mathrm{S}$ phase (16). The $p 53$ gene is a tumor suppressor gene that has been revealed to be correlated with human tumors. It is a cyclin-dependent gene and has been implicated in cell proliferation (15). A previous study demonstrated that cells expressing wild-type $p 53$, following irradiation, become locked in the G1 phase, likely due to the abnormal expression of cyclin D (15). Proliferating cell nuclear antigen (PCNA), an intra-nuclear polypeptide synthesized or expressed only in proliferating cells, serves a role in the regulation of the cell cycle. PCNA is primarily expressed during the S, G1 and G2 phases, the primary phases of proliferating cells. Its expression intensity is often used as an indicator of proliferation activity and as an assessment tool in the malignancy of tumor cells. The present study revealed that, following Clock knockdown, the expression level of p53 was decreased, while the expression of cyclin DI and PCNA was increased, indicating that the proliferation ability of cells was enhanced by the inhibition of Clock.

Downregulation of Clock in breast cancer cells may promote extracorporeal cell migration and the present study investigated the molecular drivers of this phenomenon. During the course of the study, it was revealed that the expression of IQGAPI was increased following Clock knockdown. IQGAPI is an actin scaffold protein that has been implicated in the migration of cancer cells, and it harbors a direct E-cadherin binding site. Consequently, epithelial-mesenchymal transition (EMT)-associated protein assessment revealed that cell adhesion-associated proteins, CD44 and E-cadherin, were increased by knockdown of Clock in 4T1 cells, indicating that Clock, through IQGAPI, may regulate cell-adhesion proteins to influence cell motility.

Previous reports have supported a role for IQGAP1 in the regulation of the cytoskeleton (though actin and tubulin), impacting migration, invasion and fission of colorectal cells. It has also demonstrated regulatory action on vascular endothelial growth factor, a regulator of endothelial cell migration $(17,18)$. IQGAP1 has also been documented to bind to and regulate the localization of Dia 1, another factor of cell migration. Finally, IQGAP1 has been reported to promote the migration and invasiveness of E-cadherin-mediated homologous cells, 


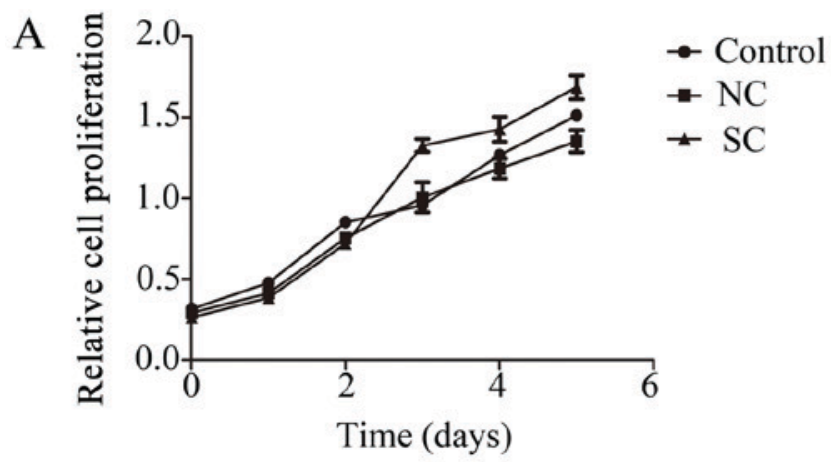

B

Control

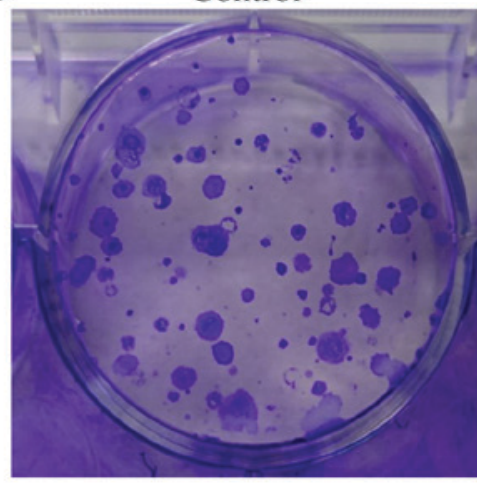

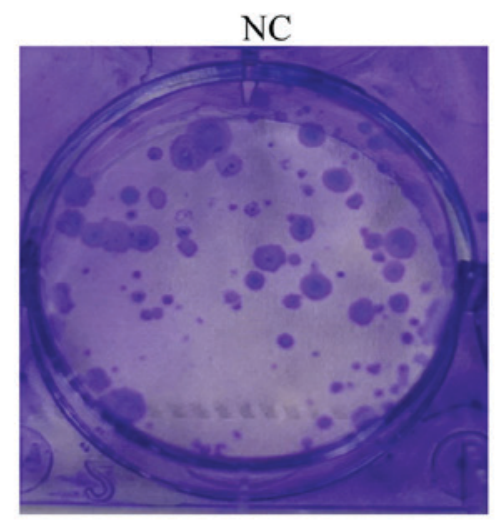

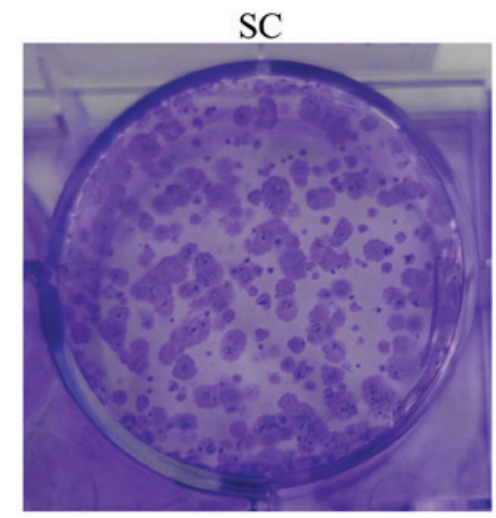

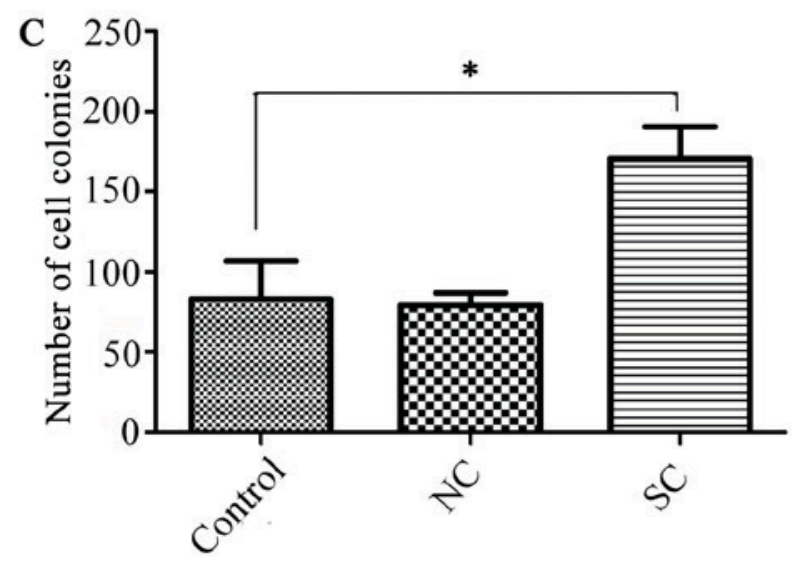

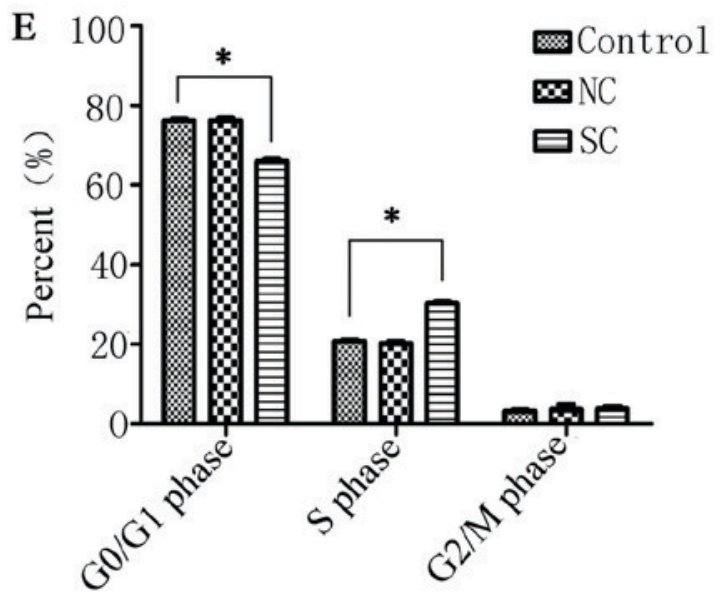

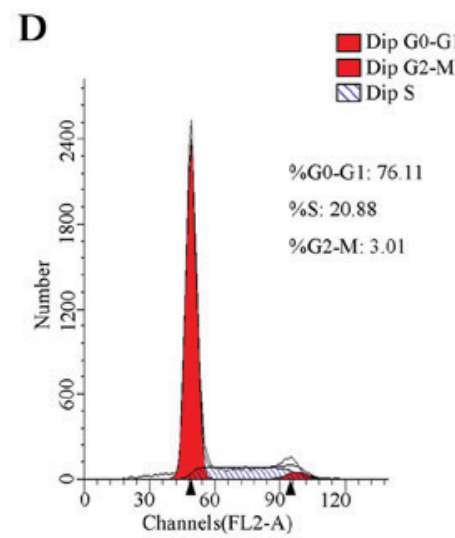

Control

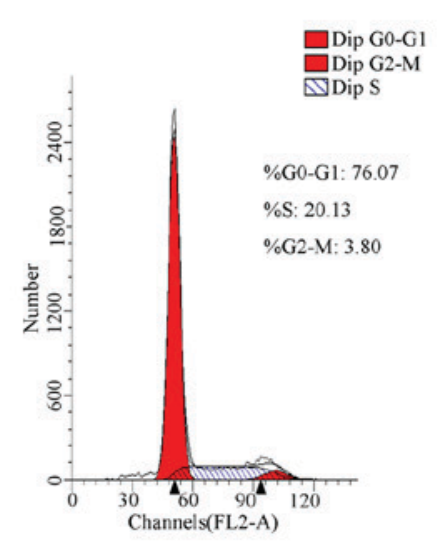

NC

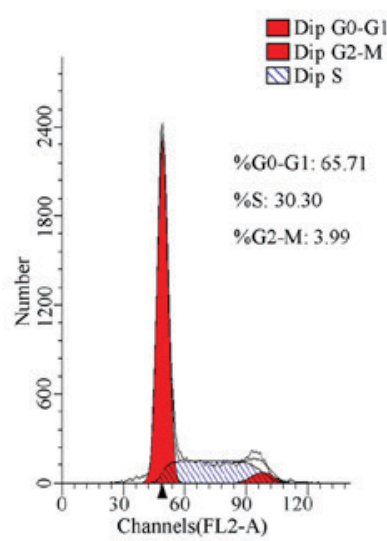

SC

Figure 2. Clock knockdown enhanced 4T1 cell proliferation. (A) Cell Counting Kit-8 assay demonstrated that silencing of Clock expression enhanced the proliferation of 4T1 cells. (B) Colony formation of 4T1 cells. (C) Quantification of the colony formation assay. (D) Cell cycle distribution was analyzed by flow cytometry. (E) Quantitative analysis of the cell cycle distribution. Data are presented as the mean \pm standard deviation of three assays. ${ }^{*} \mathrm{P}<0.05$ vs. control. Clock, circadian locomotor output cycles kaput; NC, negative control (4T1 cells transfected with lv-GFP-Puro NC virus); SC, 4T1 cells transfected with lv-shRNA-Clock virus. 
A

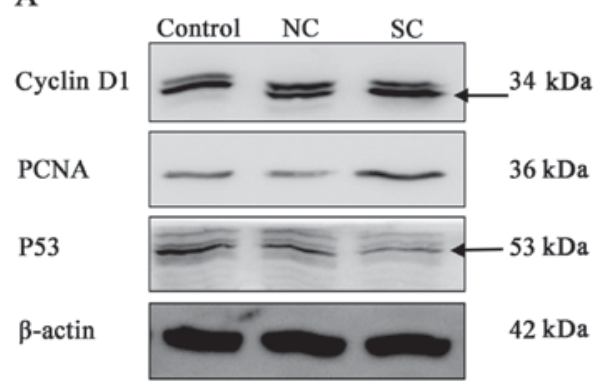

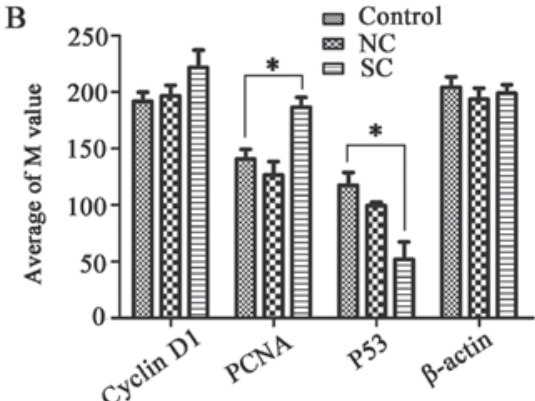

Figure 3. Association between the expression of Clock and proliferation related protein in 4T1 cells. (A) Western blot analysis of cyclin D1, PCNA and P53 expression in 4T1 cells. (B) Quantification of the western blot analysis. Data are presented as the mean \pm standard deviation of three assays. "P<0.05 vs. control. Clock, circadian locomotor output cycles kaput; PCNA, proliferating cell nuclear antigen; p53, tumor protein p53; NC, negative control (4T1 cells transfected with lv-GFP-Puro NC virus); SC, 4T1 cells transfected with lv-shRNA-Clock virus.

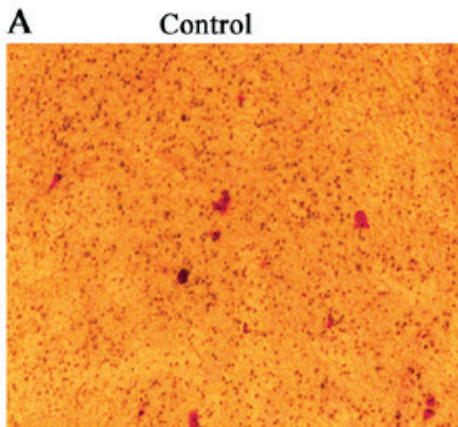

B

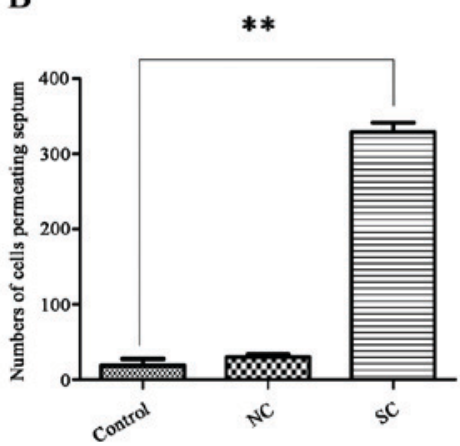

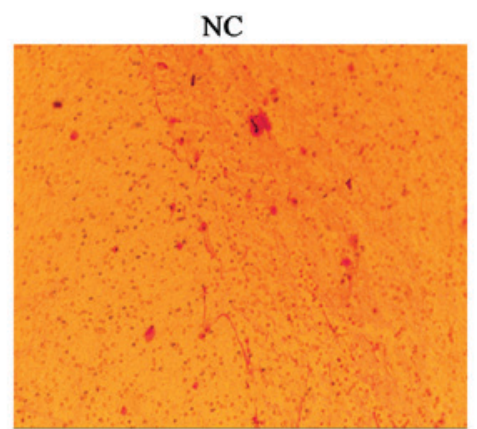

$\mathrm{C}$

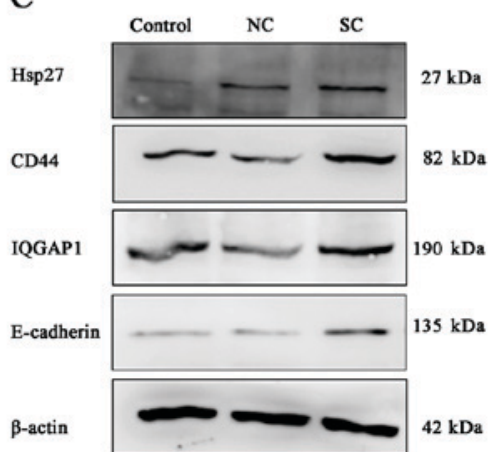

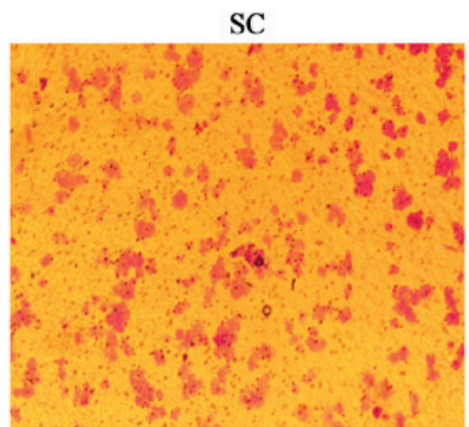

D

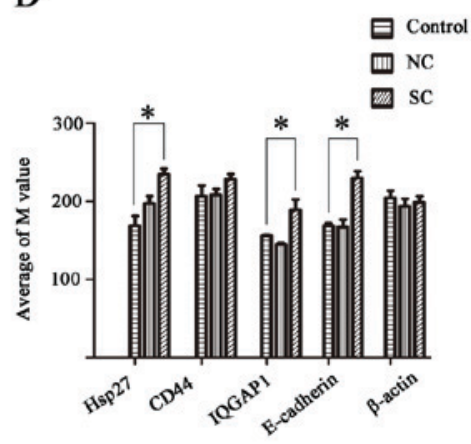

Figure 4. Effects of Clock knockdown on cell migration. (A) Transwell assay revealed that silencing of Clock expression enhanced the migration of 4T1 cells. Cells were stained with crystal violet on the underside of the chamber (magnification, x100). (B) Quantification of the Transwell assay. (C) Western blot analysis of E-cadherin, IQGAPQ, CD44 and Hsp27 expression in 4T1 cells. (D) Quantification of the western blot analysis data. Data are presented as the mean \pm standard deviation of three assays. ${ }^{*} \mathrm{P}<0.05$ and ${ }^{* *} \mathrm{P}<0.01$ vs. control. Clock, circadian locomotor output cycles kaput; NC, negative control (4T1 cells transfected with 1v-GFP-Puro NC virus); SC, 4T1 cells transfected with 1v-shRNA-Clock virus; IQGAP1, IQ motif containing GTPase activating protein 1; CD44, cluster of differentiation 44; Hsp27, heat shock protein 27.

regulating cell adhesion and plasma-membrane-mediated transposition (19-21).

The present study revealed that the expression of CD44 and E-cadherin is regulated by Clock. E-cadherin, a well-known EMT marking molecule that serves a role in maintaining cell morphology, movement and adhesion, reduced expression of which may arrest cells in their rounded state, weakening the ability of the cells to migrate and become invasive $(22,23)$. CD44, like E-cadherin, is a membrane-associated protein that regulates connectivity among heterogeneous cells, connecting tightly to the cytoskeleton and participating in the formation of cell pseudopods which are associated with cell movement and migration (24). Positive expression of CD44 has been reported to be associated with vascular infiltration and distant metastasis $(25,26)$. The present study revealed that, following knockdown of Clock, the ability of the cells to migrate was enhanced. Furthermore, Hsp27, reportedly associated with lymph node metastasis, organ metastasis, tumor size and staging (27), was similarly revealed to be upregulated by the inhibition of Clock.

In conclusion, the results of the present study suggested that the downregulation of Clock may promote the proliferation and migration of mouse breast cancer cells. Clock may affect the expression of the adhesion protein, E-cadherin, on cell membranes, likely through regulation of the expression of the skeletal-associated protein, IQGAP1. Collectively, the 
pathway affects the proliferation and migration ability of breast cancer cells. Clarifying and understanding the function and mechanisms of Clock is an important step toward elucidating the therapeutic potential of Clock and other circadian rhythm factors in cancer and other biological rhythm disorders. The present study sheds light on an important but elusive epidemiological association between circadian rhythm dysregulation and cancer.

\section{Acknowledgements}

Not applicable.

\section{Funding}

The present study was supported by the National Natural Science Foundation of China (grant no. 31371180) and the Scientific and Technological Project of Sichuan Province (grant no. 2014SZ0193).

\section{Availability of data and materials}

The datasets generated and analyzed in the present study are included in this published article.

\section{Authors' contributions}

All authors contributed to this study, LXX, WZR, and JZ designed experiment; LXX, WSY, YSH conducted the experiments; LXX, WSY, YJJ, YH analyzed experimental results. YCL, LYY, WYH, CST, XJ and GHL participated in solving the problems in the experiment. LXX, WZR and JZ wrote the paper. All authors read and approved the manuscript.

\section{Ethics approval and consent to participate}

Not applicable.

\section{Consent for publication}

Not applicable.

\section{Competing interests}

The authors declare that they have no competing interests.

\section{References}

1. Fujioka A, Takashima N and Shigeyoshi Y: Circadian rhythm generation in a glioma cell line. Biochem Biophys Res Commun 346: 169-174, 2006.

2. Feng D and Lazar M: Clocks, metabolism, and the epigenome. Molecular Cell 47: 158-167, 2012.

3. Reppert SM and Weaver DR: Coordination of circadian timing in mammals. Nature 418: 935-941, 2002.

4. Doi M, Ishida A, Miyake A, Sato M, Komatsu R, Yamazaki F, Kimura I, Tsuchiya S, Kori H, Seo K, et al: Circadian regulation of intracellular G-protein signalling mediates intercellular synchrony and rhythmicity in the suprachiasmatic nucleus. Nat Commun 2: 327, 2011.

5. Okamura H: Suprachiasmatic nucleus clock time in the mammalian circadian system. Cold Spring Harb Symp Quant Biol 72 551-556, 2007.
6. Fu L, Pelicano H, Liu J, Huang P and Lee C: The circadian gene Period2 plays an important role in tumor suppression and DNA damage response in vivo. Cell 111: 41-50, 2002.

7. Schibler U and Sassone-Corsi P: A web of circadian pacemakers. Cell 111: 919-922, 2002.

8. Reppert SM and Weaver DR: Molecular analysis of mammalian circadian rhythms. Annu Rev Physiol 63: 647-676, 2001.

9. Chen S, Choo K, Hou M, Yeh K, Kuo S and Chang J: Deregulated expression of the PER1,PER2 and PER 3 genes in breast cancers. Carcinogenesis 26: 1241-1246, 2005.

10. Roenneberg $\mathrm{T}$ and Lucas R: Light, endocrine systems, and cancer-a view from circadian biologists. Neuro Endocrinol Lett 23 (Suppl 2): S82-S83, 2002.

11. Stevens R: Circadian disruption and breast cancer: From melatonin to clock genes. Epidemiology 16: 254-258, 2005.

12. Moser M, Schaumberger K, Schernhammer E and Stevens RG: Cancer and rhythm. Cancer Causes Control 17: 483-487, 2006.

13. Hansen J: Risk of breast cancer after night- and shift work: Current evidence and ongoing studies in Denmark. Cancer Causes Control 17: 531-537, 2006.

14. Zhou JX, Niehans GA, Shar A, Rubins JB, Frizelle SP and Kratzke RA: Mechanisms of $\mathrm{G}_{1}$ checkpoint loss in resected early stage non-small cell lung cancer. Lung Cancer 32: 27-38, 2001.

15. Rose SL and Buller RE: The role of p53 mutation in BRCA1-associated ovarian cancer. Minerva Ginecol 54: 201-209, 2002.

16. Motokura T, Bloom T, Kim HG, Jüppner H, Ruderman JV, Kronenberg HM and Arnold A: A novel cyclin encoded by a bcl1-linked candidate oncogene. Nature 350: 512-515, 1991

17. Sarparanta J, Jonson PH, Golzio C, Sandell S, Luque H, Screen M, McDonald K, Stajich JM, Mahjneh I, Vihola A, et al: Mutations affecting the cytoplasmic functions of the co-chaperone DNAJB6 cause limb-girdle muscular dystrophy. Nat Genet 44: 450-455, S451-452, 2012.

18. Hunter PJ, Swanson BJ, Haendel MA, Lyons GE and Cross JC: Mrj encodes a DnaJ-related co-chaperone that is essential for murine placental development. Development 126: 1247-1258, 1999.

19. Hayashi H, Nabeshima K, Aoki M, Hamasaki M, Enatsu S, Yamauchi Y, Yamashita Y and Iwasaki H: Overexpression of IQGAP1 in advanced colorectal cancer correlates with poor prognosis-critical role in tumor invasion. Int J Cancer 126: 2563-2574, 2010.

20. Lin DC, Zhang Y, Pan QJ, Yang H, Shi ZZ, Xie ZH, Wang BS, Hao JJ, Zhang TT, Xu X, et al: PLK1 Is transcriptionally activated by NF- $\mathrm{KB}$ during cell detachment and enhances anoikis resistance through inhibiting $\beta$-catenin degradation in esophageal squamous cell carcinoma. Clin Cancer Res 17: 4285-4295, 2011.

21. Revenu C, Ubelmann F, Hurbain I, El-Marjou F, Dingli F, Loew D, Delacour D, Gilet J, Brot-Laroche E, Rivero F, et al: A new role for the architecture of microvillar actin bundles in apical retention of membrane proteins. Mol Biol Cell 23: 324-336, 2012.

22. Song H, Li Y, Lee J, Schwartz AL and Bu G: Low-density lipoprotein receptor-related protein 1 promotes cancer cell migration and invasion by inducing the expression of matrix metalloproteinases 2 and 9. Cancer Res 69: 879-886, 2009.

23. Itoh Y and Seiki M: MT1-MMP: A potent modifier of pericellular microenvironment. J Cell Physiol 206: 1-8, 2006.

24. Alemayehu M, Dragan M, Pape C, Siddiqui I, Sacks DB, Di Guglielmo GM, Babwah AV and Bhattacharya M: $\beta$-Arrestin2 regulates lysophosphatidic acid-induced human breast tumor cell migration and invasion via Rap1 and IQGAP1. PLoS One 8: e56174, 2013.

25. Xiaoping L, Xiaowei Z, Leizhen Z and Weijian G: Expression and significance of CD44 and p-AKT in pancreatic head cancer. World J Surg Oncol 13: 334, 2015.

26. McFarlane S, McFarlane C, Montgomery N, Hill A and Waugh DJ: CD44-mediated activation of $\alpha 5 \beta 1$-integrin, cortactin and paxillin signaling underpins adhesion of basal-like breast cancer cells to endothelium and fibronectin-enriched matrices. Oncotarget 6: 36762-36773, 2015.

27. Kaigorodova EV, Zavyalova MV, Bychkov VA, Perelmuter VM and Choynzonov EL: Functional state of the Hsp27 chaperone as a molecular marker of an unfavorable course of larynx cancer. Cancer Biomark 17: 145-153, 2016.

(1) (3) This work is licensed under a Creative Commons Attribution-NonCommercial-NoDerivatives 4.0 International (CC BY-NC-ND 4.0) License. 